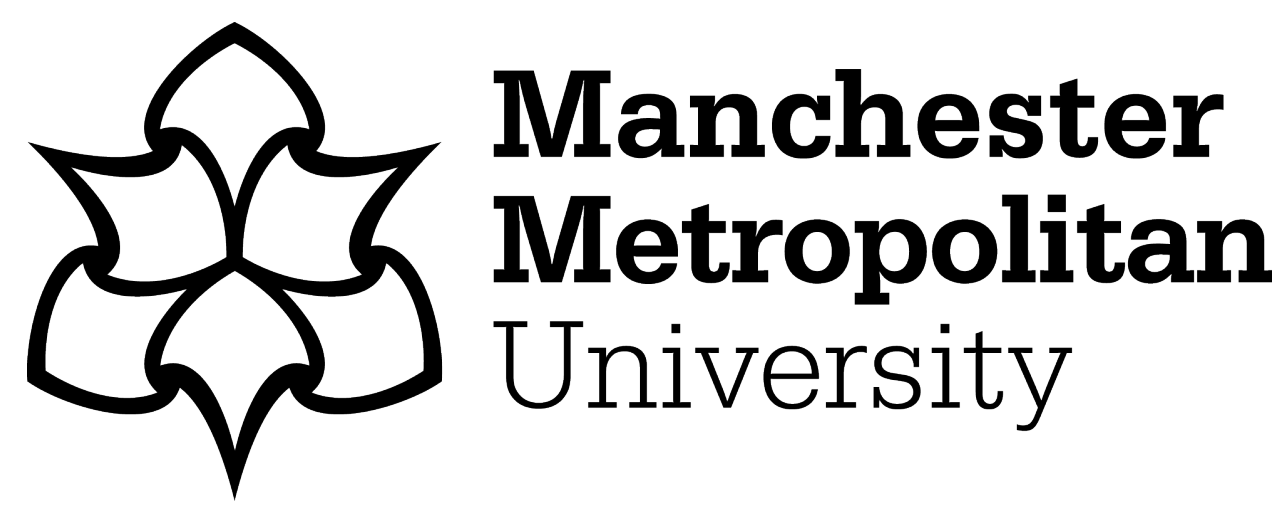

Ahsan, Md, Stoyanov, Stoyan and Bailey, Chris (2017) Data-driven prognostics for smart qualification testing of electronic products. In: IEEE 40th International Spring Seminar on Electronics Technology (ISSE), 10 May 2017 14 May 2017, Sofia, Bulgaria.

Downloaded from: https://e-space.mmu.ac.uk/624873/

Publisher: IEEE

DOI: https://doi.org/10.1109/ISSE.2017.8001010

Please cite the published version 


\title{
Data-Driven Prognostics for Smart Qualification Testing of Electronic Products
}

\author{
Mominul Ahsan, Stoyan Stoyanov and Chris Bailey \\ Computational Mechanics and Reliability Group, University of Greenwich, London, UK \\ Email: m.ahsan@greenwich.ac.uk
}

\begin{abstract}
A flaw or drift from expected operational performance in one electronic module or component may affect the reliability of the entire upper-level electronic product or system. Therefore, it is important to ensure the required quality of each individual electronic part through qualification testing specified using standards or user requirements. Qualification testing is time-consuming and comes at a substantial cost for product manufacturers. Many electronics manufacturers have access to large historical sets of qualification testing data from their products which may hold information to enable optimisation of the respective qualification procedures. In this paper, techniques from the domain of computational intelligence are applied. The development of data-driven models capable to forecast accurately and in-line the end result of a sequence of qualification tests is discussed and presented. Data-driven prognostics models are developed using test data of the electronic module by Neural Network (NN) and Support Vector Machine (SVM) techniques. The performances of the models in predicting the qualification outcomes (pass or fail) are assessed.
\end{abstract}

\section{INTRODUCTION}

The global market for the electronic product is projected to reach US $\$ 2.4$ trillion per year by 2020 [1]. This growth has led to intense competition between manufacturers to minimise the time-tomarket for their products. However, qualification testing, which is time-consuming and resourceintensive, is a major bottleneck for the quick release of electronic products to the market. Therefore, researchers and engineers have to ensure the reliability of each electronic module during qualification testing within the shortest possible time.

Qualification testing is conducted by manufacturers through measuring numerous test parameters related to the functioning of individual electronic part. The test outcome is defined either pass or fail based on the measured values fall within the specified range or not. The number of measured parameters and logical tests performed on an electronic module could vary from only a few to potentially hundreds. Therefore, an optimum qualification testing procedure is an essential requirement to minimise the long test time. The shorter test time would be highly advantageous, as it will ultimately reduce the production cost and time to introduce the product in the market. One way of reducing the test time is accurately forecasting the final test outcomes (pass/fail) using prognostics models developed by the historical data obtained from fully completed qualification tests.

Data-driven approaches such as machine learning techniques learn from historical data or training data to capture changes in test parameters monitored during qualification testing. Anomaly detection is accomplished by comparing in-situ data against healthy baseline data that is collected under several modes and load conditions under which a product is anticipated to operate [2-3]. Jaai et al. have reported a multivariate state estimation technique and executed a sequential probability ratio test to detect the onset of failure in ball grid arrays subjected to accelerated temperature cycling (ATC) tests [3]. Furthermore, a comparison performed between resistance data monitored during ATC and the baseline data to detect anomalies. Ideally, data-driven approaches in qualification testing is capable of capturing outliers in monitored data and they are effective for detecting intermittent failures while the test parameters are monitored in situ. Sohan and Lee [4] have proposed a canonical correlation analysis in order to investigate the relationship between multiple process control 
monitoring (PCM) variables and various probe bin variables of IC semiconductor. Polynomial regression is used to determine the critical values of the PCM variables that affect the performance yield. Firstly, the experiments performed involve splitting the values related to the threshold voltage in order to assure the performance yield of a device and avoid difficulties for handling all the PCM data. The PCM variables were the contact resistance, sheet resistance, and Isat_P4H as well as threshold voltage. Secondly, typical values of Vtl_P4H and Isat_P4H greater than $\pm 10 \%$ difference between the critical value and the currently-used average value should be changed in order to maximise the performance yield. The polynomial regression is useful as a methodology for identifying optimal PCM variables. The proposed method is useful for optimising the IC process to improve yield and as a problem-solving approach.

Currently, manufacturers have adopted standardsbased qualification testing [5] to ensure reliability of electronic product used in various applications. The lack of correlation between field use and test conditions promote to the inadequate qualification that causes unexpected product failure during operation and great financial losses. This is especially true for manufacturers whose product lifetimes are longer. Sometimes, harsher environments such as high temperature cause severe failures of electronic module. In general, computational techniques based on the historical test data have not been fully developed yet to reduce the qualification test time, to achieve better test outcome and to predict performance accurately. Hence, an extensive computational framework for optimising qualification test procedure, underpinned by accurate methods is required to address the challenges of predicting the test outcomes.

The aim of this study is to investigate the capability of computational intelligence algorithms such as Support Vector Machine (SVM) and Neural Network (NN) in predicting qualification test outcome of an electronic module. Prediction accuracies from both approaches are compared and reported.

\section{Methodology}

The research methodology for predicting the qualification test outcomes is presented in Fig. 1. First, a qualification test data set of an electronic module is obtained from a manufacturer to develop prognostics algorithms and optimise test procedure. The data set contains measured values of different tests for individual modules tested with a test outcome of pass and fail. The data related to pass and fail modules are separated at the data preprocessing stage. A programme has been developed in MATLAB to automatically detect and eliminate outliers from the data set. Then the measured values for individual tests are normalised to make all data within a range between 0 and 1 . The master data set is organised to develop training sets with different combinations of data related to pass and fail modules and their corresponding test outcomes. The training data sets are employed to develop machine learning models with Neural Network (NN) and Support Vector Machine (SVM). A validation or test data set (pass and fail) is also generated from the master data set to predict test outcomes from the trained models. Finally, the performance of each model is evaluated by calculating prediction error.

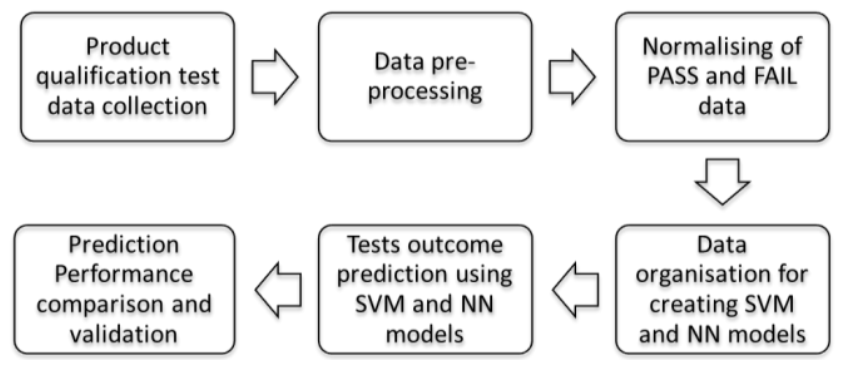

Fig. 1. Research methodology for predicting qualification tests outcome

\section{MACHINE LeARNing MethodS}

\subsection{Neural Network (NN)}

Neural Network (NN) has high efficiency in prediction purposes. Arbitrary function approximation and nonlinearity are the key significance of NN. It is well capable of establishing an effective relationship within data and showing the vibrant performance of a system.

It is essential to formulate different data sets to avoid over-fitting of data for training with the network. Training and validation are two different data sets required to run and validate the model. Fig. 2 shows the computational steps for building a $\mathrm{NN}$ model using the qualification test data of the electronic module and predicting the test outcomes. 


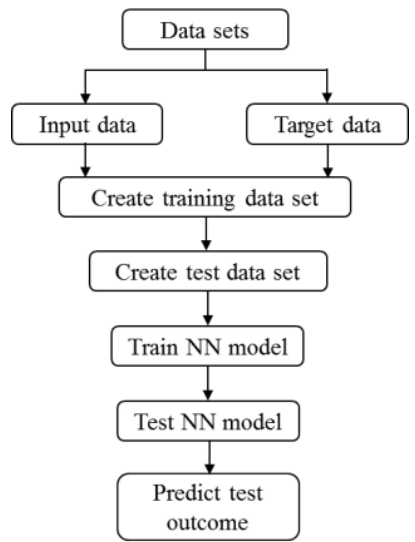

Fig. 2. Computational steps of Neural Network

Feed forward NN and Levenberg Marquardt (LM) learning algorithm are used to construct a complete $\mathrm{NN}$ model. Feed forward NN is selected for predicting the test outcomes of the qualification data. Levenberg Marquardt (LM) learning algorithm is used to train the neural network [6]. Gradient descent method and the Gauss-Newton method are combined in this network. The algorithm has the capability to solve nonlinear problems using its standard technique. Training performance is evaluated using mean squared error to simplify the construction of a network by minimising the sum of the squared errors. The sum of squares is estimated by Hessian matrix as $\mathrm{H}=\mathrm{J}^{\mathrm{T}} \mathrm{J}$, where $\mathrm{J}$ is Jacobian matrix and computed gradient as $g=\mathrm{J}^{\mathrm{T}} \mathrm{e}$ where e is network error. The Levenberg-Marquardt training algorithm is represented by Eq. 1 .

$$
X_{k+1}=X_{k}-\left[J^{T} J+\mu I\right]^{-1} J^{T} e
$$

$\mathrm{X}$ denotes connection weight, $\mu$ represents scalar combination coefficient that performs a transformation to gradient descent or Gauss-Newton algorithm and I represents as Identity matrix. Gradient descent learning rule is very common in NN training process. Error function can compute the error between the outcome of training and the desired output. This is completed by determining the sum of the squared errors of the total number of input and desired output patterns of the training set. The error function is given by Eq. 2 .

$$
\varepsilon=\sum t_{p}-f_{p}
$$

Where $t_{p}$ is the desired output and $f_{p}$ is the actual output. The target of this learning rule is to find the appropriate values that can minimise the error as illustrated in Fig. 3 [7].

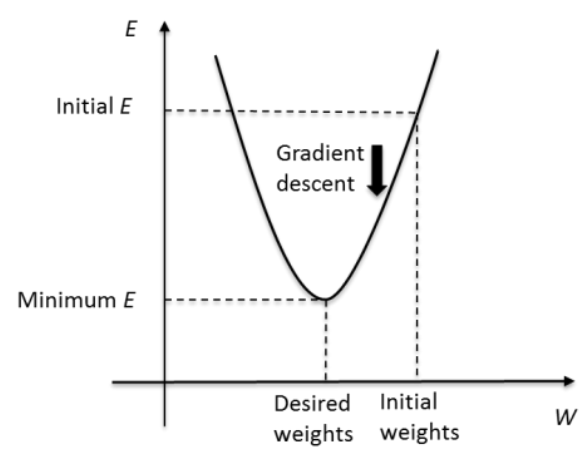

Fig. 3. Gradient descent rule of Neural Network [7]

\subsection{Support Vector Machine (SVM)}

Predictor and response data sets are required to create and train a model with SVM. Fig. 4 shows a complete process to build and validate the SVM model. After training, a new data set is required to validate the model. However, the SVM model is also capable of holding data from the training set for automatic model validation.

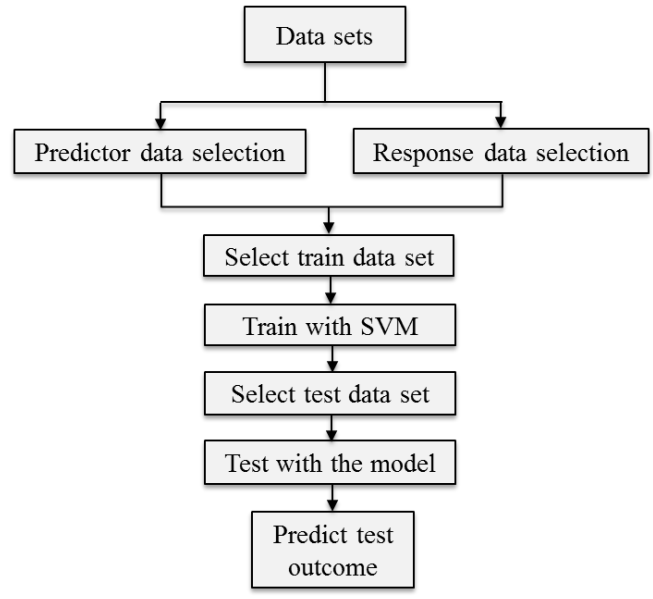

Fig. 4. Computational steps of Support Vector Machine

$\mathrm{SVM}$ is a supervised machine learning algorithm mainly used for binary classification problems that can minimise generalisation errors. It is also used for multiclass problem. The fundamental concept of this algorithm is to separate classes of objects in the data space with the use of kernel function and functional margin. Kernel functions solve nonlinear problems with higher dimension as feature space. Then the functional margin minimises the generalisation errors of classifier by finding optimal hyperplane. This hyperplane creates the finest split-up boundary between two classes (Fig. 5). To separate training data linearly, SVM technique can help to prepare the 
training data for classification into a higherdimensional space [7].

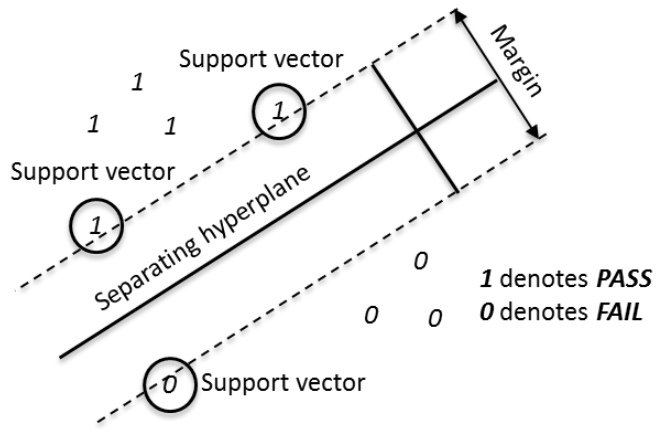

Fig. 5. Optimal hyperplane for separating data [7]

Assume $\left\{\mathrm{x}_{\mathrm{i}}-\mathrm{y}_{\mathrm{i}}\right\}(\mathrm{i}=1---\mathrm{N})$ a set of training data sample where each sample $x_{i} \in R^{n}$, $n$ is measurement of the input space which belongs to a class. The class is expressed as $\mathrm{y}_{\mathrm{i}} \in\{-1,2, \cdots \mathrm{k}\} \quad(\mathrm{k}$ denotes classification category). Hyperplane equation can be expressed by Eq. 3 .

$$
w x+b=0
$$

Where, $\mathrm{w}$ is the hyperplane vector and $\mathrm{b}$ represents a real number. Hyperplane has the ability to distribute all data according to the same level on the same side. This is how $\mathrm{w}$ and $\mathrm{b}$ maximise the margin in optimal separating hyperplane. The distance of the nearest points to hyperplane can be deducted using projection vector. Assuming equation of two boundaries $\left(\mathrm{L}_{1}\right.$ and $\left.\mathrm{L}_{2}\right)$ is $\mathrm{wx}+\mathrm{b}= \pm 1$ whereas $\mathrm{wx}_{\mathrm{i}}+\mathrm{b}=+1$ and $\mathrm{wx}_{\mathrm{i}}+\mathrm{b}$ $=-1$. To divide and fulfil maximum margin of training sample, minimising is completed under the constraint $\mathrm{y}_{\mathrm{i}}\left(\mathrm{wx}_{\mathrm{i}}+\mathrm{b}\right)>1$ which combines $\mathrm{y}_{\mathrm{i}}\left(\mathrm{wx}_{\mathrm{i}}+\mathrm{b}\right) \geq+1, \mathrm{y}_{\mathrm{i}}=1$ and $\left(w_{i}+b\right) \leq-1, y_{i}=1$. This is how linear SVM is built and can be formulated by Eq. 4 for optimisation purposes.

$$
\begin{aligned}
& \min \left\{\frac{1}{2}\|w\|^{2}\right\} \\
& y_{i}\left(w x_{i}+b\right) \geq 1, i=1, \cdots N
\end{aligned}
$$

Eq. 4 is a convex programming that is consisted of convex function and constraint. To divide and fulfil maximum margin of training sample, minimising is completed under the constraint yi $\left(\mathrm{wx}_{\mathrm{i}}+\mathrm{b}\right)>1$ which combines $\mathrm{y}_{\mathrm{i}}\left(\mathrm{wx}_{\mathrm{i}}+\mathrm{b}\right) \geq+1, \mathrm{y}_{\mathrm{i}}=1$ and $\left(\mathrm{wx}_{\mathrm{i}}+\mathrm{b}\right) \leq-1, \mathrm{y}_{\mathrm{i}}$ $=1$. Finally, optimal separating hyperplane decision function is expressed using Kuhn-Tucker optimisation theorem defined by Eq. 5, where, a* represents corresponding optimal solution [7].
$M(x)=\operatorname{sign}\left(w_{0} x+b_{0}\right)=\operatorname{sign}\left(\sum_{\mathrm{i}=1}^{\mathrm{N}} \mathrm{a}_{\mathrm{i}}^{*} y_{i}\left(\mathrm{x}_{\mathrm{i}} \mathrm{x}\right)+\mathrm{b}_{0}\right.$

\section{Demonstration Study CaSe}

\subsection{Qualification test data}

The qualification test of the electronic module contains data from different types of tests (e.g., logical, electrical etc.) and a total of 95 sequential tests are conducted for each module to determine whether it passes or fails. Measured values for each sequential test are arranged in column wise for each module in a row. However, no further test is carried out once a module is failed. Hence, no test data will be available for the module from the point of failure.

\subsection{Prognostics models}

Three different data sets for a total of 1164 modules are developed as training data sets from the master data set by varying the ratio between the no of pass and fail modules to build NN and SVM models. The ratio of no of failed and passed modules is expressed as R by Eq. 6 .

$$
\text { Ratio, } \mathrm{R}=\frac{\text { No of FAIL modules }}{\text { No of PASS modules }}
$$

The training sets contain data for 60 tests, and the outcomes are known for each module from the master data set. The models are trained to predict outcomes (fail or pass) for the validation data set. Again, a new data set is developed from the master data with 80 modules (40 pass and 40 fail) to validate the performance of the models in all cases. Table 1 shows the data arrangement for the training data sets with their corresponding ratios and the validation data set.

The neural network is comprised of three layers as input, hidden and output layers as shown in Fig. 6.

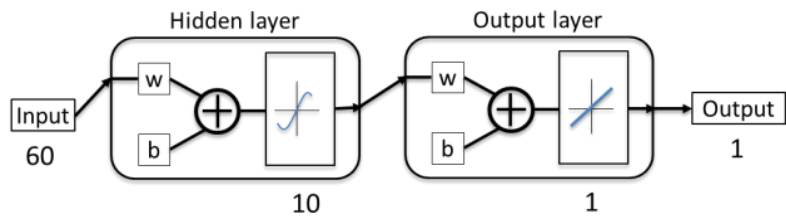

Fig. 6. Neural Network (NN) model structure.

In the NN model, data of 60 sequential test measurements for 1164 modules are considered as input and corresponding actual pass (1) and fail (0) outcomes are considered as target data. 
Table 1. Data arrangement for training and validation in SVM and NN

\begin{tabular}{|c|c|c|c|}
\hline $\begin{array}{l}\text { Machine } \\
\text { learning } \\
\text { algori- } \\
\text { thms }\end{array}$ & $\begin{array}{l}\text { Training } \\
\text { data sets }\end{array}$ & $\begin{array}{c}\text { Ratio of no } \\
\text { of fail and } \\
\text { pass } \\
\text { modules }\end{array}$ & $\begin{array}{c}\text { Valida- } \\
\text { tion } \\
\text { data set }\end{array}$ \\
\hline \multirow{3}{*}{$\begin{array}{l}\text { Support } \\
\text { Vector } \\
\text { Machine } \\
\text { (SVM) }\end{array}$} & $\begin{array}{c}582 \text { pass and } \\
582 \text { fail } \\
\end{array}$ & 1.00 & \multirow{3}{*}{$\begin{array}{c}80(40 \\
\text { pass and } \\
40 \text { fail) }\end{array}$} \\
\hline & $\begin{array}{c}764 \text { pass and } \\
400 \text { fail }\end{array}$ & 0.52 & \\
\hline & $\begin{array}{c}964 \text { pass and } \\
200 \text { fail }\end{array}$ & 0.21 & \\
\hline \multirow{3}{*}{$\begin{array}{c}\text { Neural } \\
\text { Network } \\
(\mathrm{NN})\end{array}$} & $\begin{array}{c}582 \text { pass and } \\
582 \text { fail } \\
\end{array}$ & 1.00 & \multirow{3}{*}{$\begin{array}{c}80(40 \\
\text { pass and } \\
40 \text { fail })\end{array}$} \\
\hline & $\begin{array}{c}764 \text { pass and } \\
400 \text { fail }\end{array}$ & 0.52 & \\
\hline & $\begin{array}{c}964 \text { pass and } \\
200 \text { fail }\end{array}$ & 0.21 & \\
\hline
\end{tabular}

In SVM, the same 60 sequential tests measurements for 1164 modules are used as the predictor data and corresponding actual pass (1) and fail (0) are considered as response data to train and build an SVM model (Fig. 7).

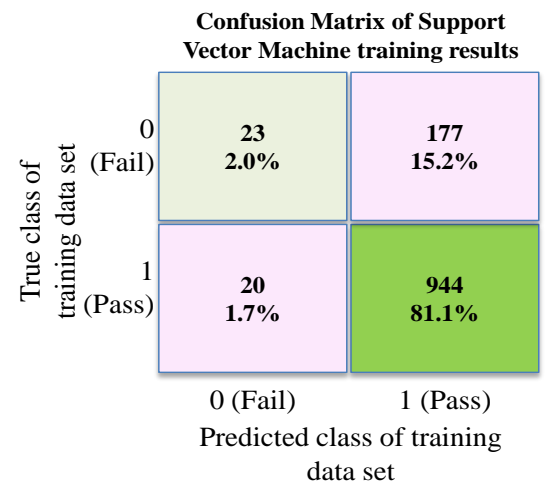

Fig. 7. Support Vector Machine (SVM) training model

\subsection{Validation}

Validation of pass/fail outcome prediction for 80 new modules (40 pass and 40 fail) is completed with the trained NN and SVM models. For both models, validations are conducted considering both data from pass and fail modules separately.

The comparison of prediction accuracies of the test outcomes between the SVM and NN models are presented in Fig. 8. Both models show high prediction accuracy since the validation is done with the pass data only. The prediction accuracies of the SVM models are gradually increasing with a decrease of the ratio possibly due to an increase of the pass data in the training sets. On the contrary, the prediction accuracies by the NN models remain constant to $100 \%$ for all the ratios.

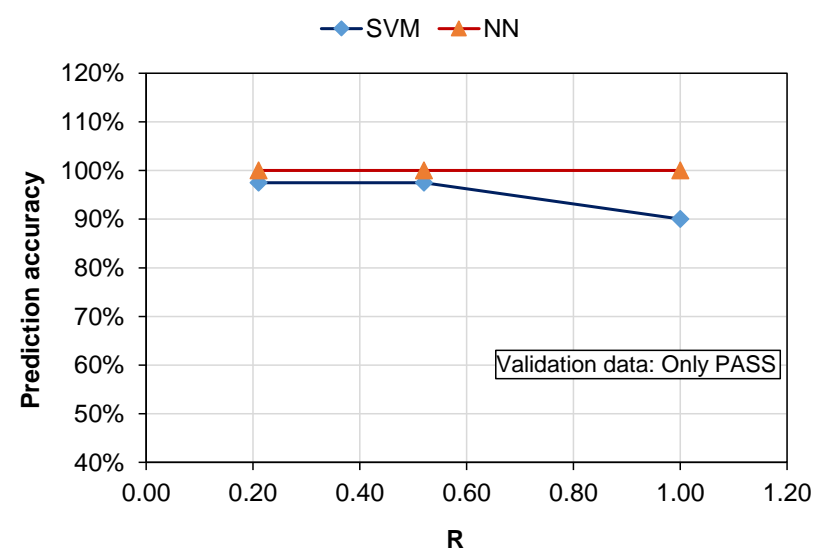

Fig. 8. Qualification test outcome prediction using SVM and NN models when validated with the pass data only

Fig. 9 demonstrates the prediction accuracies for both models after validating with the failed data only.

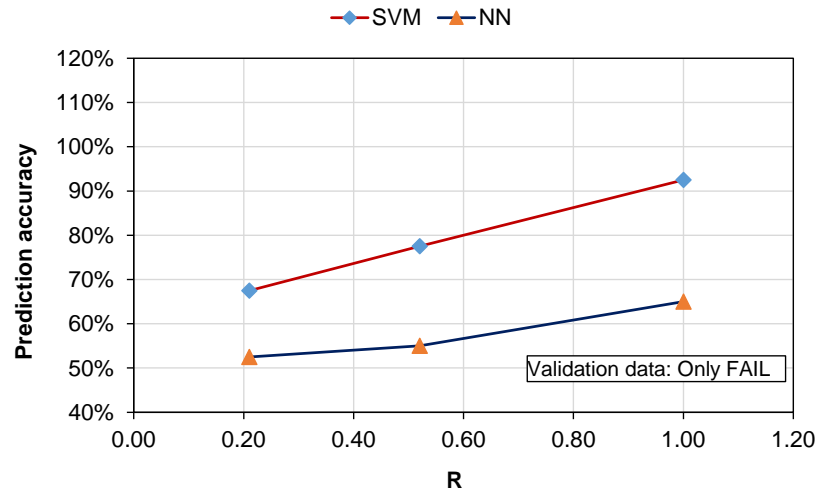

Fig. 9. Qualification test outcome prediction using SVM and NN models when validated with the fail data only

It is very clear from the figure that best prediction accuracy is achieved by the SVM model (92.50\%) when the ratio is 1 . On the contrary, NN models show significantly lower prediction accuracies compared to the SVM models. In both cases, prediction accuracy decreases with a decrease of the ratio possibly due to a reduction of fail data in the training sets.

Table 2 presents the prediction performance of SVM and NN models for validation with the pass and fail data separately. Overall, it seems SVM models can predict with better accuracy than the NN models for the all ratios. 
Table 2. Prediction performances comparison between SVM and NN models

\begin{tabular}{|c|c|c|c|}
\hline $\begin{array}{l}\text { Machine } \\
\text { learning } \\
\text { algorithm }\end{array}$ & $\begin{array}{c}\text { Ratio of no } \\
\text { of fail and } \\
\text { pass } \\
\text { modules } \\
\end{array}$ & $\begin{array}{l}\text { Prediction } \\
\text { with PASS } \\
\text { validation } \\
\text { data only } \\
\end{array}$ & $\begin{array}{l}\text { Prediction } \\
\text { with FAIL } \\
\text { validation } \\
\text { data only } \\
\end{array}$ \\
\hline \multirow{3}{*}{$\begin{array}{l}\text { Support } \\
\text { Vector } \\
\text { Machine } \\
\text { (SVM) }\end{array}$} & 1.00 & $90.00 \%$ & $92.50 \%$ \\
\hline & 0.52 & $97.50 \%$ & $77.50 \%$ \\
\hline & 0.21 & $97.50 \%$ & $67.50 \%$ \\
\hline \multirow{3}{*}{$\begin{array}{c}\text { Neural } \\
\text { Network } \\
(\mathrm{NN})\end{array}$} & 1.00 & $100.00 \%$ & $65.00 \%$ \\
\hline & 0.52 & $100.00 \%$ & $55.00 \%$ \\
\hline & 0.21 & $100.00 \%$ & $52.50 \%$ \\
\hline
\end{tabular}

Fig. 10 shows an overall scenario for predicting the qualification test outcomes. The models can predict the test outcomes (pass or fail) with a small number of sequential test data (60) instead of conducting all 95 tests. This will reduce the test time in production and time to supply the products to the customers.

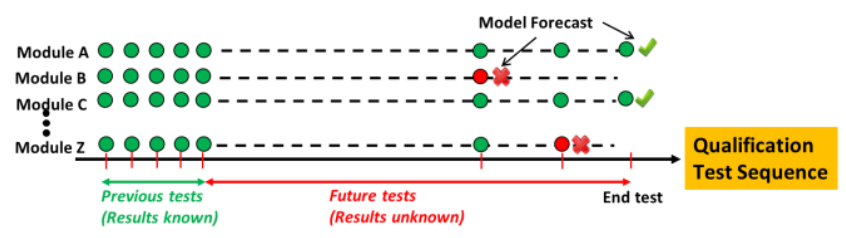

Fig. 10. Prediction scenario for qualification test outcome

\section{Conclusions}

This study develops a computational approach for predicting qualification test outcome of an electronic module using historical test data obtained from a manufacturer. It has been demonstrated that datadriven predictive models based on Neural Network and Support Vector Machine can predict the qualification test outcomes, which can reduce the overall test times by reducing the number of tests at the production stage. For both models, the prediction accuracy varies with the ratio of number of failed and passed modules used in the training data sets. However, the variation is much higher when the measurement data for only failed modules is used in the validation data set. Prediction accuracies are improved when the ratio increases or the number of failed modules in the training data sets increases.
Overall SVM models show better prediction accuracies over the $\mathrm{NN}$ models for all ratios.

Further studies will be conducted with different data arrangements both in the training and validation data sets to gain further improvement in the prediction performance of the SVM and NN models. The effect of larger training data sets with different ratios and the variation in the number of tests in the training and validation data sets will be explored.

\section{ACKNOWLEDGEMENT}

The authors thank Microsemi Corporation (www.microsemi.com) for giving access to the actual qualification test data sets and for the valuable discussions on the subject of this work.

\section{REFERENCES}

[1] Pecht. M., Shibutani, T., Kang, M., Hodkiewicz, M. and Cripps, E., A fusion prognostics-based qualification test methodology for microelectronic products, Microelectronics Reliability, 2016, vol. 63, pp. 320-324.

[2] Kumar, S., Dolev, E. and Pecht, M., Parameter selection for health monitoring of electronic products, Microelectron. Reliab. 2010, vol. 50, no. 2, pp. 161168

[3] Jaai, R., Pecht, M. and Cook, J., Detecting failure precursors in BGA solder joints, Proc. Annual Reliability and Maintainability Symposium, Fort Worth, TX, USA, January 26-29, 2009, pp. 100-105

[4] Sohn, S.Y. and Lee, S.G, "Probe test yield optimisation based on canonical correlation analysis between process control monitoring variables and probe bin variables, Expert Systems with Applications, 2012, 39 pp. 4377-4382.

[5] Jonathan, M. Souza, C.R.D., Silva A.B., Guazzi, M., Paul Chase, Bensimhon, D., Peberdy, M. A., Ashley, E., West, E., Cahalin, L.P., Forman, D. and Ross Arena, R., A neural network approach to predicting outcomes in heart failure using cardiopulmonary exercise testing, International Journal of Cardiology, 2014, Vol. 171, no. 2, pp 265-269.

[6] Engelbrecht, A. B. (2002) Computational IntelligenceAn Introduction. WILEY, 2002, pp. 1-288.

[7] Jian. S., Song, L., Linlan, L., Liqin. Z. and Gang. H, "Research on Link Quality Estimation Mechanism for WSN Based on SVM," Chinese Journal of Electronics, 2017, vol. 26, no. 2, pp. 377-384. 